\title{
Does high density-low pressure etching depend on the type of plasma source?*
}

\author{
N. Hershkowitz, ${ }^{\dagger}$ J. Ding, ${ }^{\text {a) }}$ R. A. Breun, R. T. S. Chen, J. Meyer, ${ }^{\text {b) }}$ and A. K. Quick \\ Engineering Research Center (ERC) for Plasma Aided Manufacturing, University of Wisconsin-Madison, \\ Madison, Wisconsin 53706-1687
}

(Received 10 November 1995; accepted 24 January 1996)

Etching of $\mathrm{SiO}_{2}$ with $\mathrm{CF}_{4}$ in three types of high density-low pressure $\left(5 \times 10^{11} \mathrm{~cm}^{-3}, 1-10\right.$ mTorr $)$ etch tools: electron cyclotron resonance (ECR), inductively coupled (ICP), and helicon (HRF) is described. Although the physical processes that produce the plasma in the three types of sources are quite different, the etch rate processes are identical when viewed from the wafer sheath boundary. Measurements demonstrate that if sufficient fluorine is present, the etch rate limiting step depends only on the ion energy flux to the wafer, rather than on the details of the chemical species. Etch rate control depends only on the wafer bias power. Experimental results are device independent so the etch rate in high density-low pressure plasma sources does not depend on the plasma source power. Major differences in tool etch rate characteristics are more likely determined by tool wall material (and wall chemistry) and tool geometry rather than the physical process that is used to produce the plasma. (C) 1996 American Institute of Physics. [S1070-664X(96)92805-3]

\section{INTRODUCTION}

Semiconductor fabrication depends on the ability to etch patterns with high accuracy into layers of semiconductor and dielectric materials. Patterns are normally transferred to photoresist applied to the substrates. Before 1985, chemical etching provided a convenient way to etch the patterns but that technique has the disadvantage that, for most materials, it is isotropic. For example, chemical etching etches the walls of a trench as fast as the bottom of a trench. This limits the etching to only those structures separated by more than their final depth. Around 1985, this condition was no longer satisfied as structure separations were reduced to below 1 $\mu \mathrm{m}$ while depths remained at the order of $1 \mu \mathrm{m}$. Isotropic chemical etching became unsatisfactory and was replaced by plasma etching. In plasma etching, ion acceleration across sheaths at the semiconductor wafer provides directed ion energies at the wafer surface. Anisotropic plasma etching, which provides vertical walls on trenches and vias (holes), etc., is based on the anisotropy in the ion distribution function at the wafer being etched. ${ }^{1}$

Capacitively coupled plasma etching tools have been the mainstay of the semiconductor industry since the mid 1980's. Such tools normally operate at "high pressure" (the order of 100 mTorr) and "low" density $\left(10^{10} \mathrm{~cm}^{-3}\right)$. Capacitive tools apply rf with potential variations of several hundred volts between the electrodes. Sheaths at insulating wafers provide a rectification of the plasma loss current which causes the wafers to "self-bias" to potentials a fraction of half the peakto-peak voltage. This results in sheath potential drops of up to several hundred volts. The combination of high voltage and high pressure results in thick collisional sheaths $(1 \mathrm{~cm}$ or more). Most of the application of such tools has depended on the development of recipes for the appropriate gas mixtures.

\footnotetext{
*Paper 1IA3, Bull. Am. Phys. Soc. 40, 1644 (1995).

†'Invited speaker.

${ }^{a}$ Current address: Applied Materials, Santa Clara, California.

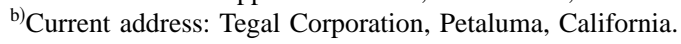

While this approach has been very successful, it has two potential problems. Ion scattering in the thick sheath gives rise to ion velocity perpendicular to the normal to the wafer surface. This places a limit on etch anisotropy (which can be overcome by side wall passivation in some cases). The high voltage drop across the sheath can result in radiation damage produced by energetic ions. In addition to anisotropy, the other major issues are etch rate uniformity and selectivity, the ability to etch one material (e.g., $\mathrm{SiO}_{2}$ ) and simultaneously not etch other materials such as photoresist or $\mathrm{Si}$. Currently, uniformity the order of $\pm 2 \%$ is desired over wafers $150 \mathrm{~mm}$ (or larger) in diameter.

The minimum semiconductor structure pattern size has continued to halve in size every five years and is approaching $0.25 \mu \mathrm{m}$ in 1996 while depths have remained the order of $1 \mu \mathrm{m}$. The limitation on anisotropy introduced by perpendicular sheath ion scattering and the radiation damage associated with the high sheath potential drop appear to limit high pressure capacitive discharges to linewidths $\geqslant 0.3 \mu \mathrm{m}$. The search for a solution to this problem has led to the development of "high" density, "low" pressure etch tools, where the plasma potentials are much lower than in capacitive tools, with plasma densities $10^{11} \rightarrow 10^{12} \mathrm{~cm}^{-3}$, and neutral pressures 1 mTorr $<p<10$ mTorr. A variety of different types of plasma sources can produce high density-low pressure plasmas. These include: electron cyclotron resonant (ECR), inductively coupled (ICP), helicon (HRF), and multidipole enhanced capacitive "triode" sources. In this paper we consider the questions: Does high density-low pressure etching depend on the type of plasma source? The various sources have many differences, but do the differences matter? In particular, are there any important differences? To address this question we consider $\mathrm{SiO}_{2}$ etching using $\mathrm{CF}_{4} / \mathrm{Ar}$ in three different types of high density-low pressure experimental etch tools based on ECR, helicon, and inductive sources. 


\section{HIGH DENSITY-LOW PRESSURE SOURCE CHARACTERISTICS}

High density-low pressure etch tools operate with plasma densities in the range $10^{11} \rightarrow 10^{12} \mathrm{~cm}^{-3}$ with neutral pressures $1 \mathrm{mTorr}<p<10$ mTorr corresponding to neutral densities $n_{0} \approx 3 \times 10^{12} \rightarrow 3 \times 10^{14} \mathrm{~cm}^{-3}$. Plasma potentials are much lower than in capacitive tools $(<100 \mathrm{~V}$ in comparison to $250-750 \mathrm{~V}$ in capacitive tools). All employ a separate rf source (often at $13.56 \mathrm{MHz}$ ) to "self-bias" insulating substrates to negative potentials (relative to the plasma) the order of tens of volts and use similar wafer chucks to hold, cool, and bias the wafers. The combination of high plasma density and low sheath potentials results in sheaths that are much thinner than those in capacitive discharges and because of the low neutral pressures, much thinner than ion-neutral collision lengths. The reduced ion energy at the wafer can result in reduced radiation damage. The three types of etch tools employ similar chemistries to achieve the same goals. Electron temperatures $T_{e}$ are comparable in the three types of tools, the order of $2 \rightarrow 4 \mathrm{eV}$ and increase in a similar way with decreasing neutral pressure.

High density sources are normally operated with plasma densities in the range $10^{11} \rightarrow 10^{12} \mathrm{~cm}^{-3}$. This range is not their highest possible density and much higher plasma densities can be achieved with helicon and ECR sources. However, the present generation of photoresists used to pattern wafers limits ion power $/ \mathrm{cm}^{2}$ to values which correspond to ion densities in the range less than a few times $10^{12} \mathrm{~cm}^{-3}$. Higher source densities leads to photoresist reticulation caused by overheating.

The plasmas and neutral species in high density-low pressure etch tools are quite similar but there are some obvious differences. These include the operating frequency and the presence or absence of $B$ fields. The details of the electron velocity distribution functions are quite different because of the different electron heating mechanisms. The three types of tools also provide the plasma with different combinations of conducting and insulating boundaries; although this is very tool specific and not necessarily generic to the type of tool. For identical input power, flow rate and pressure, the relative concentrations of ion, and neutral species are somewhat different. Differences exist, but do these differences matter?

Although it is obvious, it needs to be pointed out that semiconductor processing depends on the plasma and neutral parameters at the wafer surface and only indirectly on the parameters in the bulk plasma. In high density-low pressure plasmas, sheaths are collisionless, i.e., thin relative to device dimensions and all relevant collision lengths. Almost all of the plasma potential variation in a high density-low pressure etch tool occurs across boundary sheaths and the potential difference across the wafer sheath boundary determines the ion energy at the wafer surface. This potential difference is largely determined by the rf applied to self-bias the wafer. Sheaths at other boundaries influence ion sputtering and ion induced desorption at those boundaries. This may influence the formation of polymers on those boundaries, recombination of molecules on the boundaries and the overall tool neutral gas chemistry which can be a big effect.
It also appears to be obvious that plasma etch rates will be tool independent in plasma etch tools if they are operated with identical plasma and neutral parameters at the plasmawafer sheath boundary. However, it is not clear that this condition can ever be satisfied even for relatively simple etching systems. The major complication is the presence of many ion and neutral species, which includes species in the ground and metastable states. While many of the species can be characterized with available diagnostics, many cannot. Our experiments suggest that identical etching can be achieved by matching a limited number of plasma and neutral parameters at the plasma-wafer sheath boundary. Once these parameters are identified, it is not necessary to determine the others, except perhaps in very general ranges.

\section{CHARGED PARTICLES AT WAFERS}

The "self-bias" potential at insulating wafers, accelerates ions to the wafer and reflects most of the plasma electrons. Wafers normally float electrically with each point receiving no net current over the rf cycle. The details of the electron distribution function are more important in determining the chemistry than it is in direct interaction with the wafer.

The variation of the electron temperature $T_{e}$ with neutral pressure is determined by the balance of plasma production with plasma loss. Plasma loss can be written as a Bohm current to the appropriate loss surface. For ionization from the tail of a Maxwellian electron distribution function, this can be written as

$$
n_{e}\left\langle\sigma_{i}\left(T_{e}\right) v_{e}\right\rangle n_{0} V \approx n_{e} c_{s} A_{\text {loss }},
$$

where $c_{s}=\sqrt{T_{e} / m_{i}}, v_{e}$ is the electron thermal velocity, $n_{e}$ is the plasma density, $\sigma_{i}$ is the ionization cross section, $n_{0}$ is the neutral density, $V$ is the plasma volume, $A_{\text {loss }}$ is the plasma loss area and \langle\rangle denotes an average over the electron velocity distribution function. The plasma density $n_{e}$ drops out of Eq. (1). Rearranging Eq. (1) shows that $T_{e}$ is a function of only neutral pressure and geometry and is not a function of input power. $T_{e}$ depends on the geometry of the etch tool and the gas chemistry rather than on the details of the mechanism of electron heating. Therefore, $T_{e}$ versus pressure in ICP, HRF, and ECR etch tools are similar. Differences can be attributed to deviations from Maxwellian distribution functions.

Etch rates are sensitive to the ion energy $E_{i}$ at the wafer surface:

$$
E_{i}=e\left(-V_{s b}+\Phi_{p}\right),
$$

where $V_{s b}$ is the wafer self bias voltage and $\Phi_{p}$ is the plasma potential far from the wafer. The ion transit time through the wafer sheath is the order of $1 / \omega_{p i}$, where $\omega_{p i}$ is the ion plasma frequency. For high density-low pressure plasmas with densities of $10^{11} \rightarrow 10^{12} \mathrm{~cm}^{-3}$, the choice of $13.56 \mathrm{MHz}$ as the frequency for rf self-bias gives ion sheath transit times comparable to or shorter than the rf period, giving rise to a spread in ion energies which depends on the phase of the self bias $\mathrm{rf}$ at the time the ion reaches the sheath edge.

Ions also pick up approximately $T_{e} / 2$ in falling through the presheath that connects the sheath to the plasma (see Fig. 


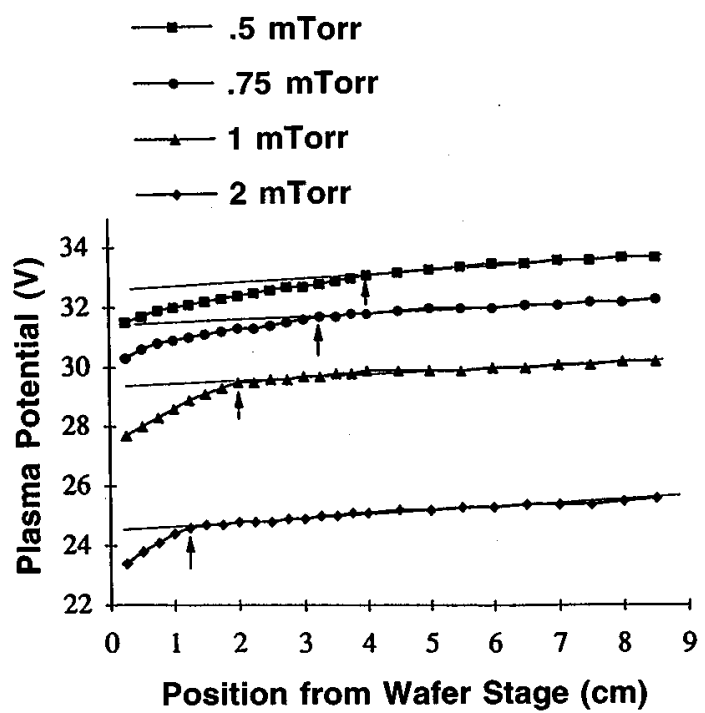

FIG. 1. Plasma potential versus position near the wafer in the ECR tool for several neutral pressures. The arrows indicate the edge of the presheath.

1). ${ }^{2}$ The presheath does not make a significant contribution to the ion energy at the wafer but is important in determining the etch anisotropy. The etch rate anisotropy (i.e., the ratio of etch rate perpendicular to the surface to the etch rate parallel to the surface), depends on $E_{\perp i}$, the ion energy parallel to the wafer surface. Ion motion in the presheath is essentially collisionless. The presheath characteristic length has been found to be equal to the smaller of the ion-neutral charge exchange collision length or the wafer to device boundary separation. Inelastic ion scattering in the presheath can transfer up to $T_{e} / 2$, into the direction transverse to the sheath. Therefore,

$$
E_{\perp i} \leqslant \text { presheath energy } \approx \frac{T_{e}}{2} .
$$

In low density-high pressure etch tools, $E_{\perp i}$ comes from ion-neutral scattering in the much thicker sheaths in such plasmas. Then presheath scattering is not important. In low pressure tools, most of the ion energy is acquired in accelerating across the plasma wafer sheath. In high density-low pressure tools, the sheaths are thin, ${ }^{3}$ the order of $\left(E_{i} / T_{e}\right)^{3 / 4} \lambda_{\mathrm{D}}$, where $\lambda_{\mathrm{D}}$ is the electron Debye length. The etch anisotropy also depends on the source chemistry because it is sensitive to the deposition or redeposition of "passivating layers" such as polymers on side walls which are not easily removed by ion sputtering.

\section{OXIDE ETCHING WITH $\mathrm{CF}_{4}$}

We chose etching of $\mathrm{SiO}_{2}$ and $\mathrm{Si}$ with $\mathrm{CF}_{4} / \mathrm{Ar}$ as a model etching system. Representative chemical reactions involving neutral species originating from $\mathrm{CF}_{4}$ are given in the Appendix. It is apparent that a large variety of species are present. It is generally believed that $\mathrm{SiO}_{2}$ etching is provided by atomic fluorine. Among the etch products are $\mathrm{SiF}_{4}, \mathrm{SiF}_{2}$, $\mathrm{SiOF}_{2}, \mathrm{CO}, \mathrm{CO}_{2}$, and $\mathrm{COF}_{2}$. Many ion species are also present.

We have employed many in situ diagnostics of plasma and neutral species. These include Langmuir probes, multi-

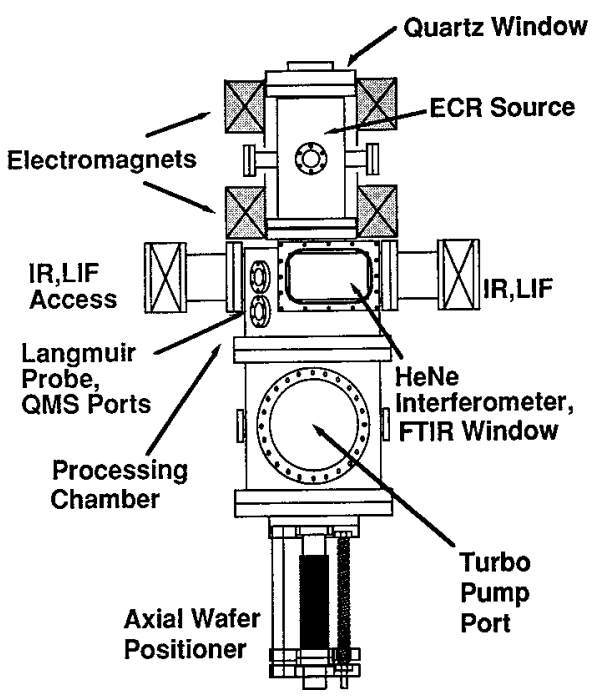

FIG. 2. Schematic description of the ECR etch tool.

pass infrared absorption, Fourier transform infrared absorption (FTIR), optical and vacuum ultraviolet emission (VUV), mass spectrometers, laser induced fluorescence (LIF), microwave interferometry, and x-ray emission.

Experiments were carried out with three experimental etch tools shown schematically in Figs. 2-4. Each was designed to provide much better diagnostic access than commercial etch tools. In all three tools, uniform $\mathrm{SiO}_{2}$ films on $\mathrm{Si}$ were etched. Etch rates were determined in situ from interference fringes from the front and back side of the $\mathrm{SiO}_{2}$ films using a $\mathrm{He}-\mathrm{Ne}$ laser, and ex situ using a profilometer and an ellipsometer.

The ECR tool operated at $2.45 \mathrm{GHz}$ (see Fig. 2) was based on a commercial ASTeX ECR source with a stainless

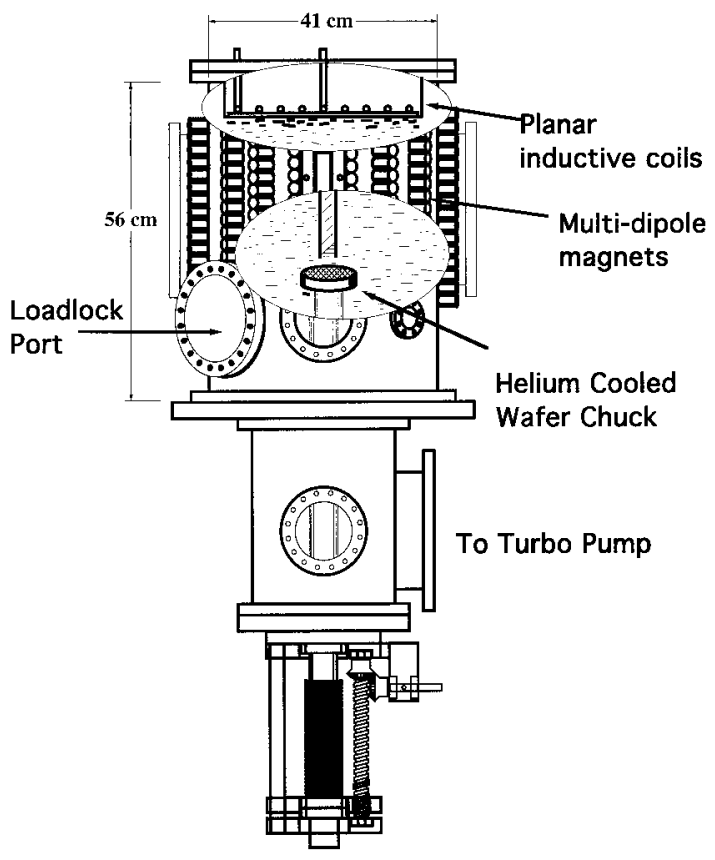

FIG. 3. Schematic description of the magnetically confined inductively coupled plasma (MCICP) etch tool. 


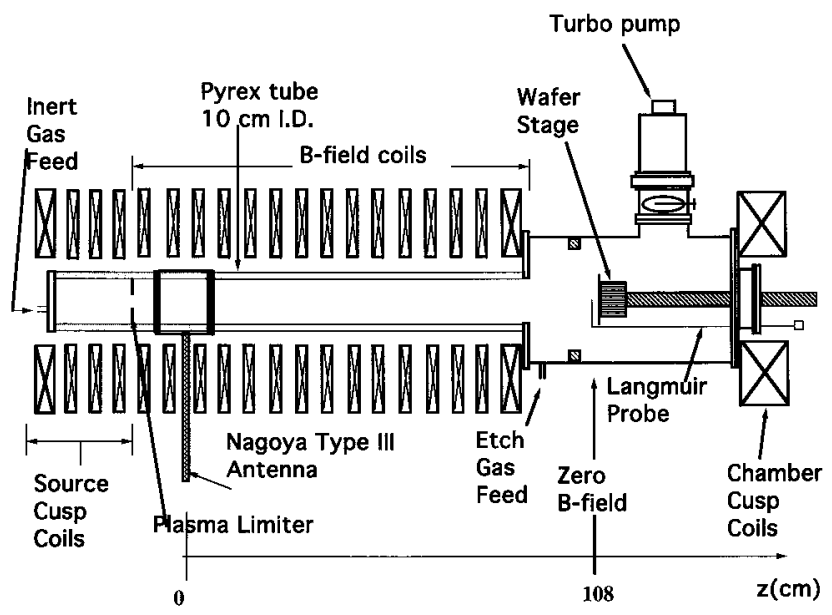

FIG. 4. Schematic description of the HRF etch tool.

steel vacuum chamber and an anodized aluminum liner in the cyclotron resonance region. Electrons were heated near the location of the electron cyclotron resonance. The magnetic field was produced by two magnetic mirror coils with the resonant magnetic field of $875 \mathrm{G}$ in the "source region" between the coils. Plasma etching took place downstream where the magnetic field was approximately $100 \mathrm{G}$. By adjusting the magnetic field and plasma density at the wafer, etch rate uniformity of approximately $\pm 2 \%$ was achieved over 4 in. diameter wafers.

The ICP (shown in Fig. 3) employed a planar spiral coil operated at $13.56 \mathrm{MHz}$, a quartz window and a stainless steel chamber. It differed from commercial etching tools by the presence of a multidipole magnetic field on the cylindrical walls which provided improved radial confinement. Electrons are heated by inductive electric fields within a skin depth located within a few centimeters of the quartz plate. By adjusting the spiral coil geometry, etch rate uniformity of approximately $\pm 2 \%$ was achieved.

The helicon source (see Fig. 4) produces energetic electrons by a combination of wave electron trapping in the antenna near field, Landau damping and collisional heating. The uniform magnetic field (which can be varied up to 1.5 $\mathrm{kG}$ ) was produced by a set of pancake magnets. Helicon waves were excited by a Nagoya-type III coil operated at 13.56 MHz. The plasma produced in the uniform magnetic field region is very nonuniform both axially and radially. Uniform etch rate at a $150 \mathrm{~mm}$ wafer was achieved by reversing the current in the coils behind the wafer producing a magnetic cusp field with the wafer placed in the zero $B$ field region. Etch rate uniformity of $\pm 2 \%$ was achieved.

Experimental $\mathrm{SiO}_{2}$ etch rate data obtained in the ECR tool are graphed in Fig. 5. These show the etch rate generally increases with power but increases and then decreases with neutral pressure. Viewed this way the data appear to be quite complicated. The confusion can be clarified by identifying equal etch rate contours on a graph of fluorine concentration vs ion energy flux (see Fig. 6). ${ }^{4}$ The fluorine concentration was determined with argon actinometry. ${ }^{5}$ The ion energy flux was determined by measuring the ion saturation current to a probe near the wafer.

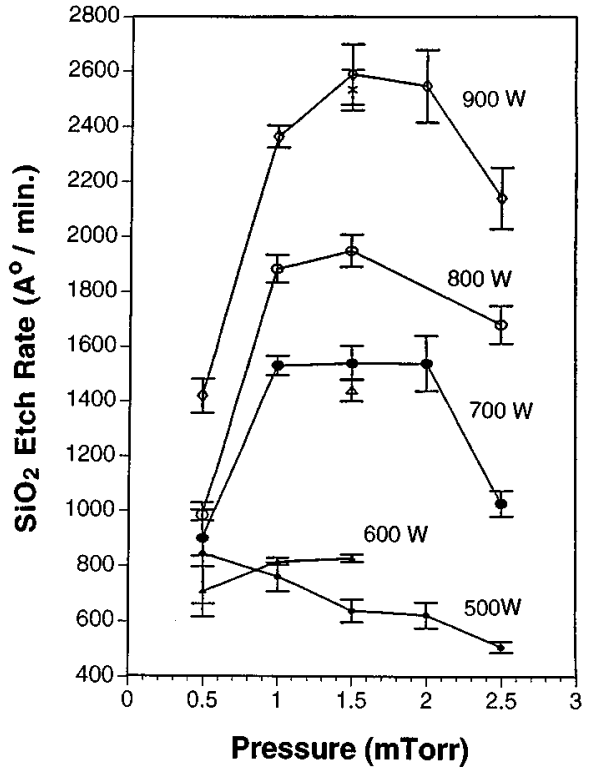

FIG. 5. $\mathrm{SiO}_{2}$ etch rate in the ECR tool versus pressure as a function of power.

All of the studies employing $\mathrm{CF}_{4}$ found the $\mathrm{SiO}_{2}$ etch rate $R$ can be described by ${ }^{4}$

$$
R \approx \frac{K_{e s} J_{i} E_{i}}{1+A_{s}\left(J_{i} E_{i} / J_{e}\right)},
$$

where $E_{i}=$ ion energy at the wafer, $J_{e}=$ etching species flux, $J_{i}=$ ion particle flux, and $K_{e s}$ and $A_{s}$ are constants. For $\mathrm{CF}_{4}$ etching of $\mathrm{SiO}_{2}$, the etching species is $F$ and the ion flux can be provided by any positive ion. Equation (4) can be derived by assuming the etch rate is proportional to the product of the ion energy flux and the surface coverage of the reacting neutrals and is also proportional to the product of the neutral flux and the bare area. ${ }^{5}$ The data presented in Fig. 6 are

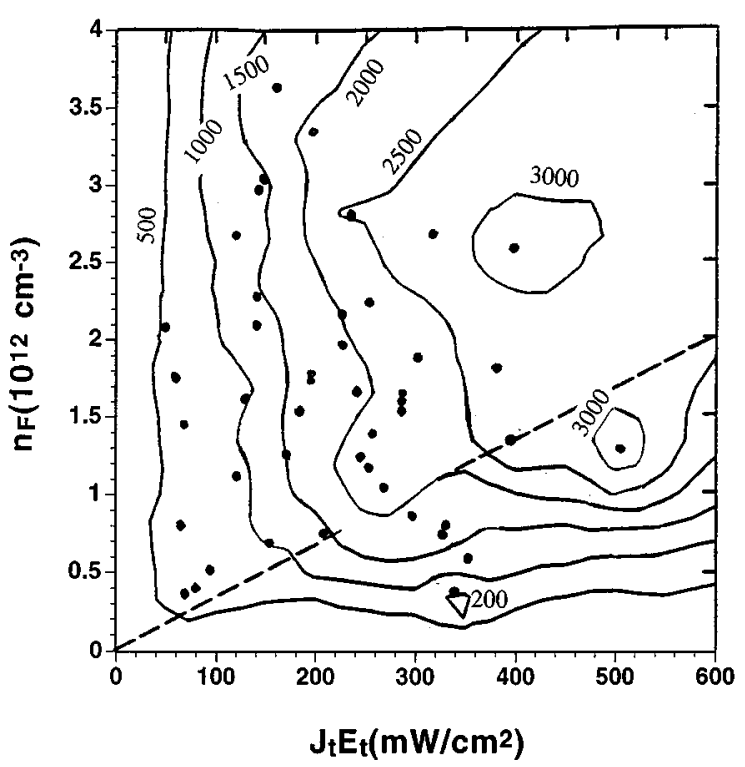

FIG. 6. Contour plot of $\mathrm{SiO}_{2}$ average etch rate $(\mathrm{A} / \mathrm{min})$ in the ECR tool as a function of ion energy flux to the wafer surface and the $F$ atom density. 


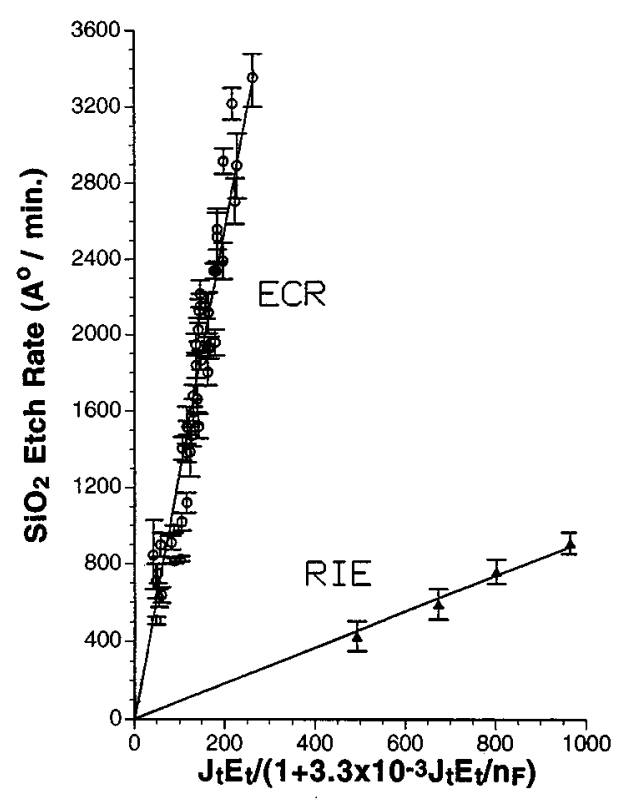

FIG. 7. ECR etch rate data $(500-900 \mathrm{~W}, 0.5-2.5$ mTorr, self-bias voltage: $25-100 \mathrm{~V})$ and RIE data $(700-1500 \mathrm{~W}, 37 \mathrm{mT})$ fit to Eq. (4). $J_{i} E_{i}$ is in $\mathrm{mW} / \mathrm{cm}^{2}$ and $n_{F}$ is in $10^{12} \mathrm{~cm}^{-3}$.

graphed versus $R$ calculated from Eq. (4) in Fig. 7. $\mathrm{SiO}_{2}$ etch rate was also measured in a capacitive "Reactive Ion Etch" (RIE) tool. The etch rate data in the RIE tool are included in Fig. 7. These data are also fit to Eq. (4), but with a much smaller value of the constant $K_{e s}$. For the ECR tool $K_{e s}=13$ while for the RIE tool $K_{e s}=0.93$.

The difference in high density-low pressure and RIE etch rates can be understood by including the effects of polymer deposition on the wafer. Assuming polymer deposition is also proportional to ion energy flux and making similar assumptions as those made to derive Eq. (4) gives ${ }^{6}$

$$
R \approx \frac{K_{e s} J_{i} E_{i}\left[1-C_{s p}\left(J_{d} / J_{e}\right)^{2}\right]}{1+A_{s}\left(J_{i} E_{i} / J_{e}\right)},
$$

where $J_{d}=$ deposition species flux and $C_{s p}$ is a constant. For $\mathrm{CF}_{4}$ etching, the deposition species appears to be $\mathrm{CF}_{2}$. Our measurements find that the ratio $J_{d} / J_{e}$ is small (the order of $10^{-2}$ ) for $\mathrm{CF}_{4}$ etching of $\mathrm{SiO}_{2}$ in high density-low pressure tools we considered but that it is significant $(\approx 2.7)$ in etching experiments carried out in the RIE tool.

As written, Eqs. (4) and (5) do not depend on the type of etch tool and only depend on parameters at the wafer surface. This suggests the constants in Eqs. (5) and (6) should be universal and tool independent. A comparison of etch rates in the ECR, HRF, ICP, and RIE tools is given in Fig. 8. It is apparent that Eq. (5) fits data from all four tools with one set of coefficients. This is a remarkable result since the details of the four types of sources are quite different.

Because of the small value of $J_{d} / J_{e}$, the coefficient $C_{s p}$ is unimportant to the fits to the high density-low pressure data. On the other hand, it is important for RIE data. It is also seen on Fig. 8 that the appropriate choice of $C_{s p}=0.13$ allows data from an RIE tool to fall on the same line with $K_{e s}=13$.

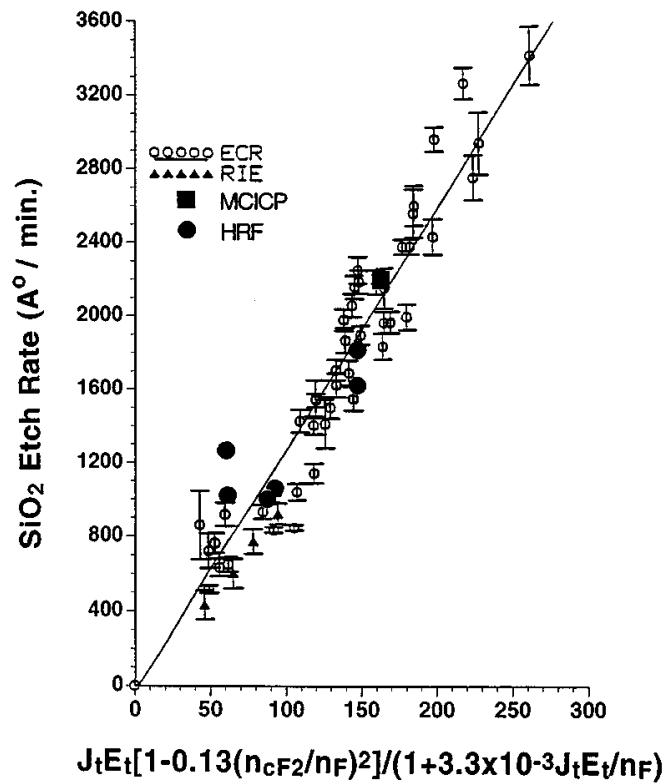

FIG. 8. Etch rate data from the ECR, HRF, MCICP, and RIE tools [given in Fig. 7, fit to Eq. (5)].

The uniformity of both etch rate and anisotropy depend on the uniformity of: $n_{e}, \phi_{p}, T_{e}$, wafer chuck-cooling, free radicals, and self-bias across the wafer surface. Equation (5) says we only need to know: $E_{i}, n_{e}, T_{e}, n_{F}, n_{\mathrm{CF}_{2}}$. We do not need to know about all of the neutral species. In addition, we only need to know the electron density $n_{e}$ rather than the individual densities of each of the ion species.

Equation (5) does not depend on the electron energy distribution function (EEDF) or the electron velocity distribution function (EVDF). Figure 8 shows that etch rate does not depend on the type of tool or on the presence or absence of a $B$ field.

We have argued that plasma parameters only matter at the wafer. During wafer etching, it is not possible to determine most parameters at the wafer surface but it is often relatively easy to monitor some parameters far from the wafer. It is important to establish how far is too far. This means we must consider the scale lengths of the various parameters.

For charged particles, the smallest scale length is given by the electron Debye length $\lambda_{\mathrm{D}} \equiv \sqrt{\epsilon_{0} T_{e} / n e^{2}}$. For high density plasmas, the Debye length is small (the order of $3 \times 10^{-3}$ $\mathrm{cm})$. The Debye length determines the sheath dimensions so it is apparent that most of the potential drop occurs in the immediate vicinity of the wafer and does not depend on the particular tool.

Sheaths are connected to the plasma by presheaths in which the plasma potential changes by $\Delta \phi \approx T_{e} / 2 e$. Representative axial potential profiles measured with emissive probes in the ECR tool have been given in Fig. 1. The presheath was found to be a transition region with approximately constant electric field, which extends from the sheath at the boundary (not shown) several centimeters into the plasma. In low pressure tools, presheath lengths are the order of the ion neutral collision length. This can be the order of $1 \rightarrow 10 \mathrm{~cm}$ depending on the gas species employed. Note that the presheath is 
much thicker than the sheath. Inelastic collisions in presheaths determine the perpendicular ion energy $E_{\perp}$ which in turn plays a role in determining the etch anisotropy.

When magnetic fields are present at an angle $\psi$ with respect to the normal to the wafer, a magnetic presheath forms, with thickness ${ }^{7}$

$$
\begin{aligned}
& \text { Magnetic presheaths } \approx \frac{c_{s}}{\Omega_{c i}} \sin \psi \approx \frac{10^{3}}{B(\text { Gauss })} \\
& \times \sin \psi(\mathrm{cm}) .
\end{aligned}
$$

For example, if the angle is $10^{\circ}$ and the $B$ field is $100 \mathrm{G}$, the magnetic presheath thickness is approximately $1.7 \mathrm{~cm}$ thick, comparable to the collisional presheath.

In low pressure plasmas, electron ionization mean free paths tend to be comparable to the tool dimensions. Other inelastic mean free paths are also relatively large. On the other hand, the mean free paths for elastic collisions tend to be relatively short. This means that electron velocity distributions tend to be isotropic in velocity space and electron energy distribution functions often provide a good description of the plasma electrons. The mean free paths for neutral species are often comparable to tool dimensions.

Examination of the characteristic scale lengths for the various processes leads to the conclusion that as far as the charged-particle components of the plasma are concerned, the interaction of the wafer collisional and magnetic presheath with the tool boundaries could be important in affecting the etch rate uniformity and the etch anisotropy.

Chemical etching and anisotropy depend on the ratio of the densities of several plasma species. For example in $\mathrm{CF}_{4}$ etching, the etch rate and anisotropy depend on $n_{e} / n_{F}$ and $n_{\mathrm{CF}_{x}} / n_{F}$, where $n_{\mathrm{CF}_{x}}$ and $n_{F}$ are the concentrations of fluorine and $\mathrm{CF}_{x}$ and $x=1,2,3,4$. The ratio of concentrations can vary with the type of tool and it is tempting to attribute the differences to differences in the electron heating mechanism or to the presence or absence of magnetic fields. However, it is more likely that the differences can be attributed to interactions of neutral species with the walls.

\section{CONCLUSION}

Does high density-low pressure etching depend on the type of plasma source? Identical plasma parameters at the sheath-wafer boundary give rise to identical etching, independent of tool type. Data obtained for $\mathrm{SiO}_{2}$ etching with $\mathrm{CF}_{4}$ find that the etch rate depends on rate limiting steps which depend on the ion energy flux or the $F$ density and etch tools need only match these parameters to obtain iden- tical etch rates. Anisotropy depends on presheath scattering which provides $E_{\perp i}$ and on source chemistry, which can provide passivating layers.

The plasma physics of ICP, HRF, and ECR sources are quite different but the $\mathrm{SiO}_{2}$ etch rate by $\mathrm{CF}_{4} / \mathrm{Ar}$ plasma is insensitive to the source plasma physics and is pretty much the same in all three types of tools when operated with the same ion energy flux and $F$ concentration. These results suggest that as far as etch rate with this system is concerned, the choice of the type of etching tool depends on the experimental knobs to tune parameters, the ease of access, the ease of construction, the cost of ownership, the ownership of patents, and the availability of maintenance. It does not depend on most of the chemical reactions or the type of tool.

\section{ACKNOWLEDGMENT}

This work was supported by NSF Grant No. EEC 8721545 .

\section{APPENDIX: REPRESENTATIVE CHEMICAL REACTIONS INVOLVING NEUTRAL SPECIES FOR $\mathrm{CF}_{4}$ PLASMAS AND Si}

$\mathrm{CF}_{4} \rightarrow \mathrm{CF}_{3}+\mathrm{F}, \quad \mathrm{CF}_{4} \rightarrow \mathrm{CF}_{2}+2 \mathrm{~F}, \quad \mathrm{CF}_{3} \rightarrow \mathrm{CF}_{2}+\mathrm{F}$,

$\mathrm{CF}_{2} \rightarrow \mathrm{CF}+\mathrm{F}, \quad \mathrm{CF}_{3}+\mathrm{F} \rightarrow \mathrm{CF}_{4}, \quad \mathrm{CF}_{3}+\mathrm{CF}_{3} \rightarrow \mathrm{C}_{2} \mathrm{~F}_{6}$,

$\mathrm{CF}_{2}+\mathrm{CF}_{2} \rightarrow \mathrm{C}_{2} \mathrm{~F}_{4}, \quad \mathrm{CF}_{2}+\mathrm{F} \rightarrow \mathrm{CF}_{3}, \quad \mathrm{CF}+\mathrm{F} \rightarrow \mathrm{CF}_{2}$,

$\mathrm{C}_{2} \mathrm{~F}_{6} \rightarrow \mathrm{CF}_{3}+\mathrm{CF}_{3}, \quad \mathrm{C}_{2} \mathrm{~F}_{4} \rightarrow \mathrm{CF}_{2}+\mathrm{CF}_{2}$,

$\mathrm{F}+\mathrm{C}_{2} \mathrm{~F}_{4} \rightarrow \mathrm{CF}_{3}+\mathrm{CF}_{2}, \quad \mathrm{CF}_{2}+\mathrm{CF}_{3} \rightarrow \mathrm{C}_{2} \mathrm{~F}_{5}$,

$\mathrm{C}_{2} \mathrm{~F}_{5}+\mathrm{F} \rightarrow \mathrm{CF}_{3}+\mathrm{CF}_{3}, \quad \mathrm{CF}+\mathrm{CF}_{2} \rightarrow \mathrm{C}_{2} \mathrm{~F}_{3}, \quad \mathrm{C}_{2} \mathrm{~F}_{3}+\mathrm{F} \rightarrow \mathrm{C}_{2} \mathrm{~F}_{4}$

and

$\mathrm{Si}+\mathrm{F} \rightarrow \mathrm{SiF}, \quad \mathrm{SiF}+\mathrm{F} \rightarrow \mathrm{SiF}_{2}$,

$\mathrm{SiF}_{2}+\mathrm{F} \rightarrow \mathrm{SiF}_{3}, \quad \mathrm{SiF}_{3}+\mathrm{F} \rightarrow \mathrm{SiF}_{4}$

${ }^{1}$ D. A. Manos and D. L. Flamm, Plasma Etching: An Introduction (Academic, Boston, 1989).

${ }^{2}$ J. A. Meyer, G.-H. Kim, M. J. Goeckner, and N. Hershkowitz, Plasma Sources Sci. Technol. 1, 147 (1992).

${ }^{3}$ M. A. Lieberman and A. J. Lichtenberg, Principles of Plasma Discharges and Materials Processing (Wiley-Interscience, New York, 1994).

${ }^{4}$ J. Ding, J.-S. Jenq, G.-H. Kim, H. L. Maynard, J. S. Hamers, N. Hershkowitz, and J. W. Taylor, J. Vac. Sci. Technol. A 11, 1283 (1993).

${ }^{5}$ J.-S. Jenq, J. Ding, J. W. Taylor, and N. Hershkowitz, Plasma Sources Sci. Technol. 3, 154 (1994).

${ }^{6}$ J. Ding and N. Hershkowitz, Appl. Phys. Lett. 68, 1619 (1996).

${ }^{7}$ G.-H. Kim, N. Hershkowitz, D. A. Diebold, and M. H. Cho, Phys. Plasmas 2, 3222 (1995). 